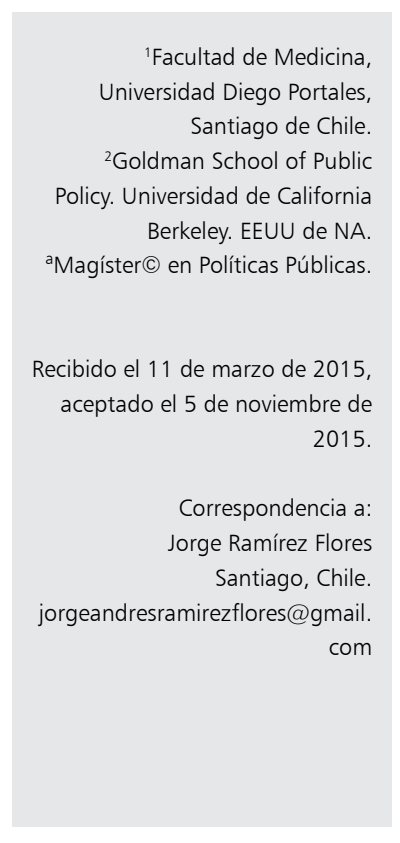

\section{Edad mínima legal de consumo de alcohol en el contexto chileno}

\author{
JORGE RAMÍREZ1, NEREIDA HELLER 2 ,a
}

\begin{abstract}
Alcohol consumption is harmful to minors. One of the most widely accepted measures for the prevention of harm associated with alcohol consumption for young people is to establish a minimum legal drinking age. This document presents the evidence available on this policy, offers a condensed analysis of its characteristics in the United States of America, describes current consumption patterns of Chilean youth, and proposes concrete solutions to be implemented.

(Rev Med Chile 2016; 144: 108-115)
\end{abstract}

Key words: Alcohol drinking, Alcoholism, Underage drinking.
$\mathrm{E}$ 1 consumo de alcohol en el mundo y en Chile es uno de los factores de riesgo más importantes para la salud de la población. La evidencia es amplia. A modo de ejemplo, se estima que el consumo de alcohol es responsable de una de cada diez muertes en el país ${ }^{1}$.

A edades tempranas, el consumo de alcohol representa un importante riesgo tanto para el desarrollo de las personas, como para el posible daño agudo a sí mismas o a terceros involucrados. Existen modelos teóricos que explican la secuencia de procesos relacionados con el beber temprano o underage drinking ${ }^{2}$ y los posibles factores de riesgo y/o protectores (específicos o inespecíficos) para iniciar este consumo en etapas más precoces ${ }^{3,4}$. La evidencia sostiene que los niños y adolescentes son más vulnerables a los efectos nocivos del alcohol de un determinado volumen de alcohol que otros grupos de edad ${ }^{5-7}$. La edad de inicio de consumo se relaciona directamente con un deterioro del estado de salud a futuro, a expensas de mayor riesgo de dependencia y abuso de alcohol ${ }^{8-12}$, de otros problemas de salud mental ${ }^{13}$, de accidentes vehiculares $^{14,15} \mathrm{y}$ de otras lesiones no intencionales ${ }^{14,16}$.

Parte del riesgo entre los jóvenes se relaciona con que una mayor proporción del alcohol es bebido durante los episodios de consumo excesivo episódico o binge drinking ${ }^{17}$. Además, los jóvenes parecen tener menos aversión al riesgo y pueden tener conductas más temerarias en estado de ebriedad.

El beber temprano se relaciona directamente con una serie de eventos negativos, y la literatura que avala esto es muy extensa ${ }^{18}$. Resulta relevante destacar que variables complejas como criminalidad también han sido relacionadas directamente ${ }^{19}$.

Cuando existe un comportamiento dañino o no deseado en la sociedad, pueden implementarse tres tipos de acciones desde el estado: uno es la regulación económica, que en este contexto implicaría algún impuesto para desincentivar el comportamiento y/o corregir externalidades negativas. Segundo, una campaña informativa, que resultaría relevante en circunstancias de asimetría de información; es decir, donde la ciudadanía no tiene la información necesaria acerca del comportamiento bajo discusión. Finalmente, existen las medidas de tipo coercitivas en las cuales el estado prohíbe explícitamente cierto comportamiento y lo supervisa con fuerza policial.

Establecer una Edad Mínima Legal de Consumo (EMLC) (o MLDA por sus siglas en inglés Minimum Legal Drinking Age) es una política de este último tipo, que además es una regulación 
social. En contraste a las regulaciones económicas, las regulaciones sociales incluyen las limitaciones al comportamiento, como las normas ambientales, las de publicidad y, en el ejemplo actual, las que especifican cuáles sustancias se pueden consumir legalmente, en qué momento y a qué edad. La EMLC refleja la creencia social que el alcohol es más dañino para los jóvenes que para los adultos, lo cual está aceptado ampliamente.

\section{Efectividad de la EMLC: el caso estadounidense}

Existen múltiples medidas de salud pública tendientes a disminuir los daños asociados al beber entre los jóvenes, de demostrada eficacia. Sin embargo, no siempre resultan efectivas al momento de aplicarse en contextos "reales" 20 . Establecer una EMLC resulta eficaz en reducir el consumo entre los jóvenes ${ }^{21}$. Gran parte de la evidencia que sostiene esta afirmación se basa en análisis de la situación histórica ocurrida en distintos estados de Estados Unidos de Norteamérica (EE. UU.). Después de la época de la prohibición total en la década de los 20, se permitió en 1933 que cada estado estableciera normas en relación con el consumo de alcohol. La gran mayoría de los estados establecieron la EMLC en 21 años. En 1971 se aprobó la Ley que redujo la edad legal para votar de 21 a 18 años de edad. Concomitantemente 30 Estados redujeron su EMLC a 18, 19 o 20.

Hacia comienzos de los ochentas sólo 14 estados todavía tenían un EMLC de 21. Sin embargo, se desarrolló un cúmulo de evidencia respecto a los accidentes fatales en adolescentes en estados con menor EMLC, por lo que el gobierno federal promulgó en 1984 una ley, que si bien no obliga a los estados, genera perjuicios económicos a los que establezcan EMLC menor a 21 años. En la práctica todos los estados acataron, y desde fines de los ochentas presentan EMLC de 21 años $^{22,23}$.

Estas etapas históricas permitieron y siguen permitiendo el estudio de diversas variables relacionadas con los cambios de la edad asignada.

Sin embargo, en sólo 5 de 50 estados la prohibición es absoluta. La gran mayoría de los estados contempla una o más de las siguientes excepciones: a) en locales privados no abiertos al público, con consentimiento de los padres; b) en locales privados no abiertos al público, sin consentimiento de los padres; c) fines religiosos; d) fines médicos (bajo prescripción médica); e) trabajos para el gobierno (agentes encubiertos); f) fines educativos (estudios culinarios); g) al reportar una emergencia médica (otro joven intoxicado) y h) en locales de venta, con consentimiento de los padres.

En cuanto a las sanciones a los comerciantes, también son variadas. Sin embargo, existe acuerdo en que la posibilidad de recibir una sanción es una característica más disuasiva que la severidad de la sanción ${ }^{24}$. Por ello se privilegian vías rápidas, como la administrativa (restricción, suspensión o revocación de licencia), por sobre persecución penal (cárcel o multas por venta a menores) o demandas civiles (respecto a la responsabilidad del comerciante por daños a terceros cometidos por menores).

Se ha visto en EE. UU. (y también en algunas regiones de Canadá), que cuando la EMLC es mayor (21 años), entre los jóvenes existe menor consumo de alcohol ${ }^{25}$, menor número de accidentes de tránsito ${ }^{22}$, menor tasa de suicidios, menor dependencia a drogas y alcohol a futuro, menor tasa de resultados negativos fetales ${ }^{26,27}$ y mayor probabilidad de disuadir eficazmente a los más jóvenes respecto del consumo de alcohol ${ }^{28}$. Desde el punto de vista contrario, y reflejando probablemente los mismos mecanismos, cuando la EMLC es menor (18 años) se presentan mayores tasas de muertes por accidentes de tránsito ${ }^{29}$, mayor riesgo de bajo peso al nacer y mayor probabilidad de muerte fetal ${ }^{30,31}$, mayor tasa de suicidios $^{32} y$ mayor tasa de conductas problemáticas al beber a futuro ${ }^{33}$.

Los efectos de este tipo de medidas se encuentran íntimamente ligados a la situación previa de la comunidad donde se implementan; es así como los resultados más alentadores se dan en medio de sociedades con mayores EMLC y/o mayor laxitud en su fiscalización, teniendo entonces más espacio para mejorar ${ }^{34}$.

Finalmente, las personas al momento de cumplir la EMLC, independientemente de su valor, 18 o 19 años en este caso, incrementan significativamente la tasa de hospitalización es por enfermedades y accidentes severos relacionados al consumo de alcohol ${ }^{35}$ y la tasa de mortalidad en hombres ${ }^{36}$. Es interesante destacar que en EE. UU. los jóvenes que ya presentan problemas al beber, apoyan la medida de disminuir la EMLC en mayor proporción que sus pares sin problemas relacionados con la bebida. Esto reflejaría la ausencia de una posición reflexiva de los primeros ${ }^{37}$. 


\section{Situación en Chile}

En Chile, se establece en 18 años la edad mínima a la que se puede comprar bebidas alcohólicas y prohíbe vender o servir alcohol a menores de edad en bares, restaurantes y similares ${ }^{38}$. Anterior a esto, la ley chilena prohibía el consumo en menores de 21 años, pero no tenía recomendaciones específicas respecto de la venta. Sin embargo, no hay prohibición explícita de ingesta de alcohol en menores de 18 años, existiendo como excepción el hecho que puedan consumir en presencia de sus padres en lugares en que se expenda comida.

El vendedor arriesga pena de prisión en su grado medio (de 21 a 40 días) y una multa de entre 3 y 10 Unidades Tributarias Mensuales (UTM) (134 y 448 dólares internacionales) $)^{39,40}$. La responsabilidad civil del comerciante no ha sido explorada en nuestro medio, probablemente asociada al sistema judicial imperante. Sin embargo, es una posibilidad que ha demostrado utilidad en EE. UU. ${ }^{41}$. Para el adolescente, el procedimiento contempla conducción a cuartel de policía y devolución a los padres o tutores. En caso de situación iterada en el tiempo, se pondrá en antecedentes al SENAME.
Según conductas autoreportadas, el porcentaje de menores de 18 años que compran alcohol es significativamente inferior al de personas adultas. Como se observa en la Figura 1, sin embargo, las cifras son preocupantes. Entre las personas que consumieron alcohol en el último año, más de $40 \%$ de menores de edad compró alcohol durante los últimos 30 días en un establecimiento para consumo fuera de las instalaciones u off-premise . $^{42}$.

Respecto a la conducción, no existen diferencias a partir de los 18 años, tanto en requisitos como en sanciones. Existe la posibilidad de conseguir licencia a los 17 años, habiendo realizado un curso obligatorio y con autorización notarial de los padres.

Debido a que la implementación de la EMLC se puede traducir en muchas y diferentes regulaciones, resulta difícil discernir con exactitud cuál de las áreas reguladas genera mayor impacto. Por ejemplo la combinación de prohibiciones de compra con sanciones de tránsito para jóvenes ${ }^{43}$. En la Tabla 1 se presenta un cuadro con 16 disposiciones respecto de menores de 21 años que se aplican en los distintos estados de EE. UU. ${ }^{44}$, y una valoración gruesa de su grado de implementación a nivel nacional en la actualidad.

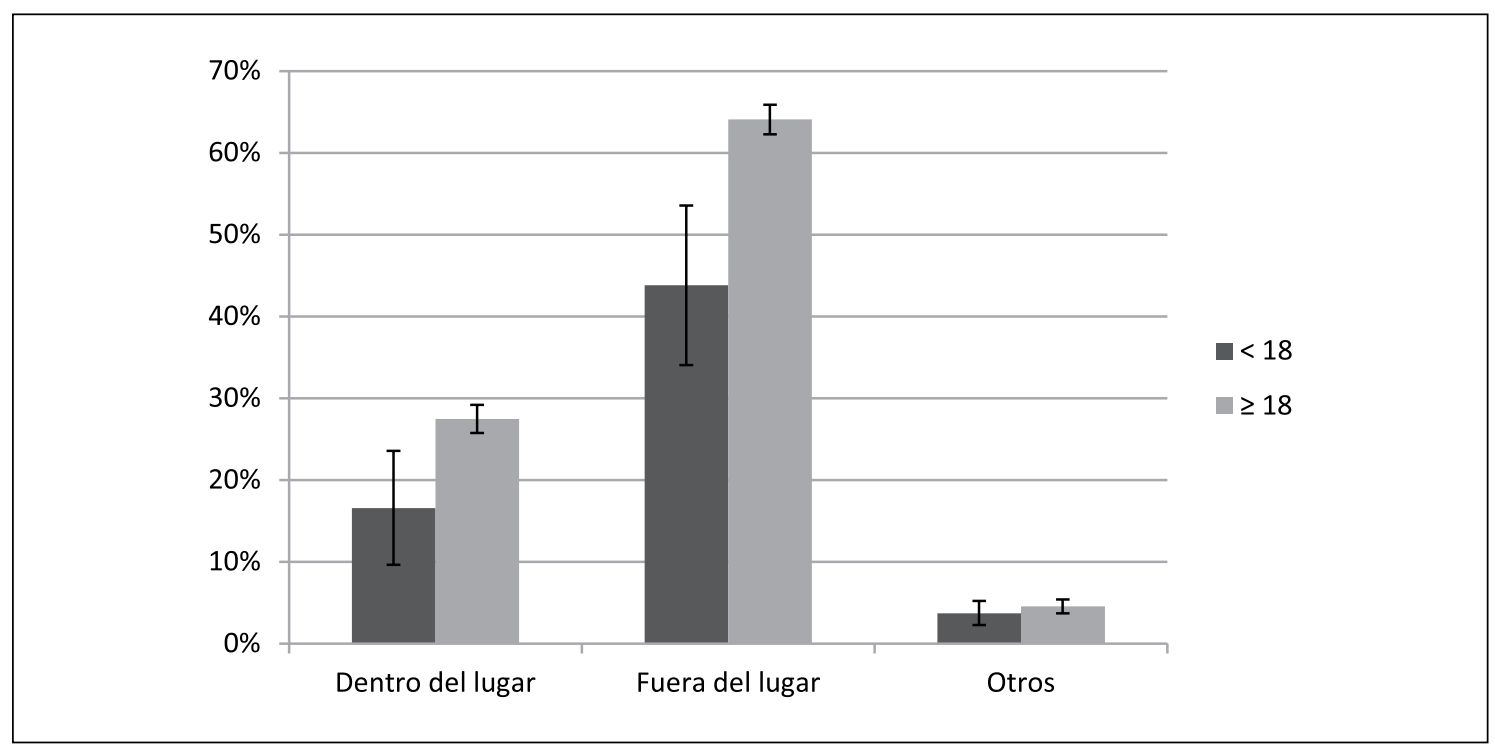

Figura 1. Porcentaje de compra de alcohol (autoreportada) durante los últimos 30 días, entre personas que consumieron alcohol en el último año, en menores y mayores de edad, según tipo de establecimiento de venta. Chile, 2012. Dentro del lugar (On-Premise): Para consumo dentro de las dependencias de venta. Incluye Bares, restaurantes, conciertos, fiestas particulares. Fuera del lugar (Off-Premise): Para consumo fuera de las dependencias de venta. Incluye botillerías, supermercados, servicentros, ferias libres, producción artesanal, clandestinos. Otros: Otros legales e ilegales. Se reportan intervalos de confianza para un 95\%. *Elaboración propia, a partir de datos del Décimo Estudio de Consumo de Drogas en Población General, SENDA 2012. 
Tabla 1. Área y contenido de distintas medidas regulatorias específicas relacionadas con el consumo de alcohol en menores en Estados Unidos de Norteamérica y su grado de implementación en Chile

\begin{tabular}{|c|c|c|c|}
\hline $\mathbf{n}$ & Área & Contenido & Grado \\
\hline 1 & Posesión & $\begin{array}{l}\text { Prohibición de posesión de bebidas alcohólicas. Se pueden aplicar excepciones } \\
\text { legales: cualquier lugar privado, cualquier residencia privada, el hogar de los } \\
\text { padres y/o tutores. }\end{array}$ & ++ \\
\hline 2 & Consumo & $\begin{array}{l}\text { Prohibición de consumo de bebidas alcohólicas. Se requiere observación de } \\
\text { la conducta, y no solamente la presencia alcoholemia positiva. Se pueden } \\
\text { aplicar excepciones. }\end{array}$ & ++ \\
\hline 3 & Compra & Prohibición de compra o intento de compra. & ++ \\
\hline 4 & Venta/regalo & $\begin{array}{l}\text { Prohibición de suministro de bebidas alcohólicas. Se pueden aplicar excep- } \\
\text { ciones. }\end{array}$ & + \\
\hline 5 & $\begin{array}{l}\text { Edad mínima para quienes } \\
\text { sirven alcohol }\end{array}$ & $\begin{array}{l}\text { Fijación de edad mínima para empleados que sirven o expenden bebidas alco- } \\
\text { hólicas en los restaurantes y similares. Pueden aplicarse excepciones. }\end{array}$ & - \\
\hline 6 & $\begin{array}{l}\text { Edad mínima para quienes } \\
\text { venden alcohol }\end{array}$ & $\begin{array}{l}\text { Fijación de edad mínima para empleados que venden alcohol en botillerías y } \\
\text { similares. Pueden aplicarse excepciones. }\end{array}$ & - \\
\hline 7 & Tolerancia Cero & $\begin{array}{l}\text { Conducir con cualquier nivel medible de alcoholes ilegal (en la práctica } \\
<0,02 \text { ). Esto puede ser diferenciado para menores. }\end{array}$ & ++ \\
\hline 8 & $\begin{array}{l}\text { Denegación de licencia de } \\
\text { conducir por infringir leyes }\end{array}$ & $\begin{array}{l}\text { Autorización para denegar otorgamiento o revocar licencia de conducir, en } \\
\text { contra de jóvenes que usaron o compraron alcohol y/o drogas ilícitas. }\end{array}$ & + \\
\hline 9 & $\begin{array}{l}\text { Registro de ventas por } \\
\text { cantidades }\end{array}$ & $\begin{array}{l}\text { Regulación que obliga a llevar registro de la venta de barriles de alcohol a } \\
\text { mayoristas y minoristas. }\end{array}$ & + \\
\hline 10 & Capacitación en servicio & $\begin{array}{l}\text { Distintos niveles de capacitación obligatoria y/o voluntaria con incentivos, para } \\
\text { personas responsables de venta de alcohol al por menor. }\end{array}$ & $+/-$ \\
\hline 11 & Uso de ID falsa & Prohibición de uso de identificación falsa explícitamente por parte de menores. & + \\
\hline 12 & Préstamo de ID & Prohibición de préstamo de identificación. & + \\
\hline 13 & $\begin{array}{l}\text { Apoyo en pesquisa de ID } \\
\text { falsa }\end{array}$ & $\begin{array}{l}\text { Disposiciones para ayudar a minoristas a identificar compradores que presen- } \\
\text { tan una identificación falsa. }\end{array}$ & - \\
\hline 14 & $\begin{array}{l}\text { Responsabilidad de } \\
\text { encargados }\end{array}$ & $\begin{array}{l}\text { Responsabilidad legal de personas por actos de menores de edad bebiendo } \\
\text { en su propiedad u otra forma de control. }\end{array}$ & - \\
\hline 15 & $\begin{array}{l}\text { Otorgamiento diferenciado } \\
\text { de licencia de conducir }\end{array}$ & $\begin{array}{l}\text { Sistema de etapas para licencias de conducir, en que se prohíbe conducción } \\
\text { nocturna y/o sin acompañante durante un período inicial. }\end{array}$ & - \\
\hline 16 & $\begin{array}{l}\text { Control estatal del } \\
\text { comercio }\end{array}$ & $\begin{array}{l}\text { Distribución a minoristas regulada por el Estado: A través del control directo } \\
\text { o del otorgamiento de patentes a privados. }\end{array}$ & + \\
\hline
\end{tabular}

- La medida no existe en Chile. +/- Existen algunas iniciativas particulares, no generalizables al país. + La medida existe; pero es pobremente fiscalizada o su forma de implementación no cumple con el objetivo de salud pública. ++ La medida existe; pero es moderadamente fiscalizada. +++ La medida existe y es adecuadamente fiscalizada. * Elaboración propia, a partir de clasificación propuesta por Fell et al., 2007.

El consumo de alcohol en menores de 18 años en Chile es común. La edad de inicio del consumo entre escolares se ha mantenido relativamente estable durante las dos últimas décadas, como se observa en la Figura 2, fluctuando alrededor de los 13 y 14 años (dependiendo del tipo de medición $)^{42,45}$, levemente menor a lo reportado en EE. UU. ${ }^{46}$.
La prevalencia se puede obtener de distintas fuentes de encuestas nacionales. En el análisis de los últimos años se observa un aumento sostenido de la prevalencia de consumo de último mes entre personas de 12 y 18 años, hasta mediados de la primera década del siglo llegando a valores cercanos a $35 \%$, para estabilizarse con posterioridad alrededor de $18 \%$, según datos obtenidos de 


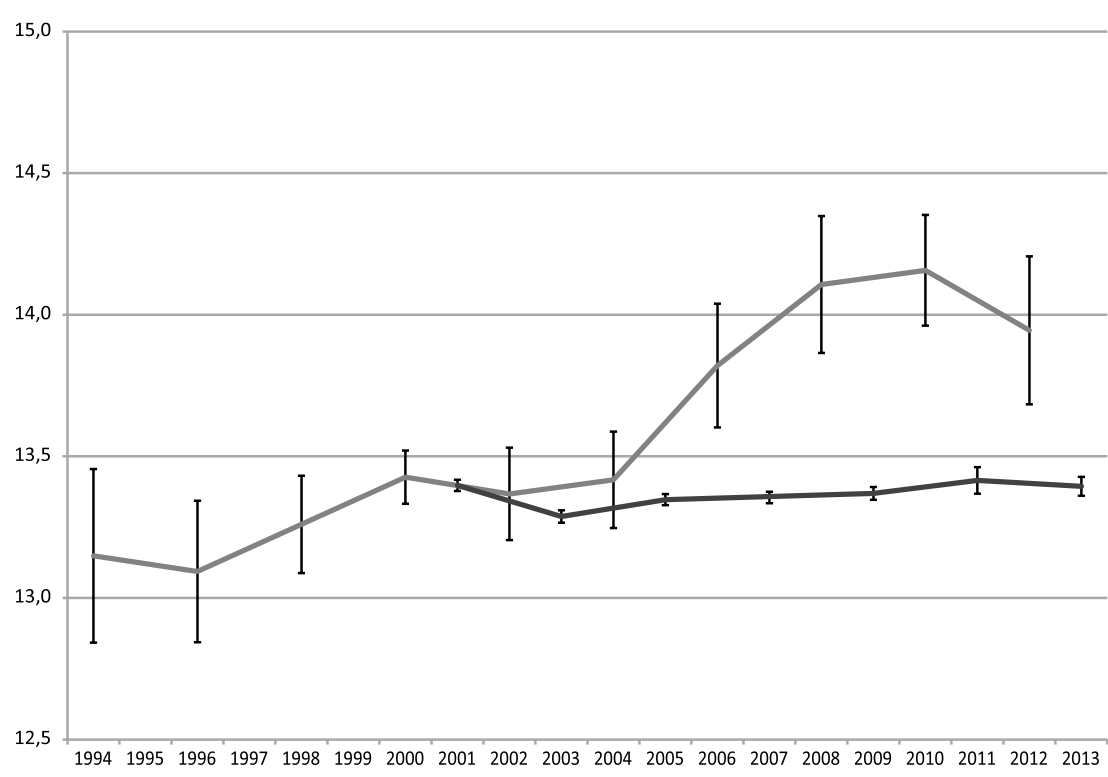

Figura 2. Edad en años de inicio de consumo de alcohol (autoreportado) en población menor de 18 años. Chile, 1994 a 2013. Línea clara, serie de Estudios en Población General, 1994-2012. Línea oscura, serie de Estudios en Población Escolar, 20012013. Se reportan intervalos de confianza para un 95\% de ambas series. Dato de 1998 en Población General es estimación lineal simple de Estudio anterior y posterior. *Elaboración propia, a partir de datos de Estudios SENDA.

población general ${ }^{42}$. Esta prevalencia es mayor a la estimada en EE. UU. por metodología similar ${ }^{46}$.

Pero no es sólo la edad de inicio o la prevalencia a determinadas edades donde recae la preocupación. Al revisar datos de medidas de riesgo en relación al consumo, 3 variables aparecen como especialmente alarmantes.

En primer término, se encuentra la frecuencia del consumo excesivo episódico, es decir la proporción de jóvenes que declara haber bebido más de 5 tragos en una ocasión, y que es un marcador del patrón de consumo ampliamente estudiado y relacionado con riesgos y daños, a corto y largo plazo. En Chile, del total de consumidores de último mes menores de 18 años, 63\% declaró haber bebido más de 5 tragos en una ocasión en el último mes $^{45}$. Se trata de una cantidad aproximada de 55 mil adolescentes.

Relacionada con la anterior, se encuentra la cantidad de alcohol puro consumido. En jóvenes entre 15 y 25 años, que consumieron alcohol la última semana, la cantidad de gramos de alcohol puro ingeridas en un día habitual de consumo es de $80 \mathrm{~g}^{47}$. Considerando que guías utilizadas a nivel nacional alertan sobre la necesidad de nunca consumir más de 4 tragos (52 aproximadamente) en una misma ocasión, esto resulta altamente preocupante $^{48}$. El dato exacto para menores de edad no está disponible oficialmente, sin embargo se debe recordar que éste es el consumo declarado en un día habitual.

Finalmente, instrumentos estandarizados, ayudan a dimensionar el problema, como el caso del AUDIT ${ }^{49}$, que relaciona el patrón de consumo del último año con la probabilidad de tener un trastorno patológico asociado al consumo de alcohol. Sobre 7\% de hombres y mujeres entre 12 y 18 presentan un puntaje elevado para este indicador ${ }^{42}$.

\section{Conclusiones}

Evidentemente, nadie duda que retrasar el inicio y disminuir el consumo de alcohol durante la juventud puede tener implicancias positivas en cuanto al daño crónico asociado al consumo de alcohol durante la vida. Existe evidencia interna- 
cional, que debe ser verificada en el contexto local, que este retraso y menor consumo se asocia a la disminución de conductas de riesgo y daño agudo provocado por el consumo de alcohol.

La segunda línea de acción de la Estrategia Nacional Sobre Alcohol del Ministerio de Salud ${ }^{50}$, respecto a la disponibilidad física del alcohol, contiene medidas que se relacionan estrechamente con la EMLC. Esfuerzos específicos están siendo llevados adelante para población joven a través de garantías explícitas para este grupo, en caso de trastornos relacionados al consumo de alcohol ${ }^{51}$.

A pesar de existir legislación sobre EMLC, esta podría estar siendo pobremente fiscalizada, como se desprende de los preocupantes datos de consumo en menores. Existe entonces la necesidad de plantear mejoras respecto de funciones esenciales de salud pública en el país para este tema, por lo que se proponen tres iniciativas concretas al respecto. El objetivo de todas ellas es aumentar la probabilidad de obtener resultados positivos mediante el uso adecuado de la EMLC frente al problema identificado: el consumo de alcohol en menores bajo la edad legal de consumo presenta un patrón de alta prevalencia y de alto riesgo en Chile.

Primero, se propone un posicionamiento público permanente de la autoridad de salud respecto al consumo y venta a menores de 18 años. Ello podría incidir a mediano plazo en la actitud y conducta de la ciudadanía frente al consumo en menores. Un ejemplo sería una campaña publicitaria centrada en los daños asociados o en las sanciones actualmente contempladas en legislación. En el contexto actual de debate respecto a las libertades personales resulta especialmente útil realizar marketing social respecto de los acuerdos sociales actualmente existentes; desde el punto de vista de salud pública, es conveniente aprovechar la conversación nacional acerca del consumo de sustancias en general para educar acerca de las repercusiones negativas del consumo de riesgo de alcohol, tomando en cuenta el grado de disponibilidad, aceptabilidad y daño de esta droga.

En segundo lugar se propone reforzar la fiscalización de la EMLC a nivel municipal. Esto permitiría aumentar la percepción de riesgo por parte de los comerciantes. Por ejemplo, establecer la ausencia de solicitud de identificación verificable para toda venta (botillerías, supermercados) e ingreso (restaurantes, discoteques) como falta grave que cause suspensión de funcionamiento y mala publicidad. La poca fiscalización de la ley no solamente deja que este comportamiento no deseable continúe, sino que genera un ambiente de desconfianza en la ley y en el estado en general. Por lo tanto, como la medida es eficaz, se deben destinar los fondos necesarios para hacerla efectiva.

Finalmente, se propone determinar un adecuado perfil del consumo de alcohol a nivel juvenil. Esto ayudaría a diseñar y focalizar otras políticas públicas destinadas a los jóvenes, sinérgicas con la EMLC. Un ejemplo sería levantar información respecto a variables no contempladas en mediciones habituales (modos de uso del alcohol, reacción a precios y sustitución por otras sustancias, georeferenciación, etc.). La necesidad de un perfil epidemiológico detallado y actualizado del consumo de alcohol en menores de edad resulta lógica para una política de salud pública destinada al comportamiento multifactorial de un grupo etario específico.

Agradecimientos. Esta investigación empleó información de los Estudios Nacionales de Drogas. El autor agradece al Servicio Nacional para la Prevención y Rehabilitación del Consumo de Drogas y Alcohol (SENDA), que depende administrativamente del Ministerio del Interior y Seguridad Pública de Chile, haberle permitido disponer de la base de datos. Todos los resultados obtenidos del estudio o investigación son de responsabilidad del autor y en nada comprometen a dicha institución.

Los autores agradecen a Álvaro Castillo, María Fernanda Toledo y Pedro Zitko por su ayuda con el análisis de datos y/o comentarios generales sobre el artículo.

\section{Referencias}

1. Castillo-Carniglia A, Kaufman JS, Pino P. Alcohol-attributable mortality and years of potential life lost in Chile in 2009. Alcohol 2013; 48 (6): 729-36.

2. Masten AS, Faden VB, Zucker RA, Spear LP. Underage drinking: a developmental framework. Pediatrics 2008; 121 Suppl 4: S235-51.

3. Zucker RA, Donovan JE, Masten AS, Mattson ME, Moss HB. Early developmental processes and the continuity of risk for underage drinking and problem drinking. Pediatrics 2008; 121 Suppl 4: S252-72.

4. Windle M, Spear LP, Fuligni AJ, Angold A, Brown JD, Pine D, et al. Transitions into underage and problem 
drinking: summary of developmental processes and mechanisms: ages 10-15. Alcohol Res Health 2009; 32 (1): 30-40.

5. Hilton ME. Demographic characteristics and the frequency of heavy drinking as predictors of self-reported drinking problems. Br J Addict 1987; 82 (8): 913-25.

6. Midanik LT, Clark WB. Drinking-related problems in the United States: description and trends, 1984-1990. J Stud Alcohol 1995; 56 (4): 395-402.

7. Makela K, Mustonen H. Relationships of drinking behaviour, gender and age with reported negative and positive experiences related to drinking. Addiction 2000; 95 (5): 727-36.

8. Grant BF. Age at smoking onset and its association with alcohol consumption and DSM-IV alcohol abuse and dependence: results from the National Longitudinal Alcohol Epidemiologic Survey. J Subst Abuse 1998; 10 (1): 59-73.

9. Grant BF, Dawson DA. Age at onset of alcohol use and its association with DSM-IV alcohol abuse and dependence: results from the National Longitudinal Alcohol Epidemiologic Survey. J Subst Abuse 1997: 9: 103-10.

10. DeWit DJ, Adlaf EM, Offord DR, Ogborne AC. Age at first alcohol use: a risk factor for the development of alcohol disorders. Am J Psychiatry 2000; 157 (5): 745-50.

11. Kraus L, Bloomfield K, Augustin R, Reese A. Prevalence of alcohol use and the association between onset of use and alcohol-related problems in a general population sample in Germany. Addiction 2000; 95 (9): 1389-401.

12. Sartor CE, Lynskey MT, Heath AC, Jacob T, True W. The role of childhood risk factors in initiation of alcohol use and progression to alcohol dependence. Addiction 2007; 102 (2): 216-25.

13. Skogen JC, Sivertsen B, Lundervold AJ, Stormark KM, Jakobsen R, Hysing M. Alcohol and drug use among adolescents: and the co-occurrence of mental health problems. Ung@hordaland, a population-based study. BMJ Open 2014; 4 (9): e005357.

14. Hingson R, Heeren T, Zakocs R. Age of drinking onset and involvement in physical fights after drinking. Pediatrics 2001; 108 (4): 872-7.

15. Hingson RW, Zha W. Age of drinking onset, alcohol use disorders, frequent heavy drinking, and unintentionally injuring oneself and others after drinking. Pediatrics 2009; 123 (6): 1477-84

16. Cherpitel CJ. Focus on: the burden of alcohol use-trauma and emergency outcomes. Alcohol Res 2013; 35 (2): 150-4.

17. USA, in The Surgeon General's Call to Action To Prevent and Reduce Underage Drinking. 2007: Rockville (MD).
18. Healey C, Rahman A, Faizal M, Kinderman P. Underage drinking in the UK: changing trends, impact and interventions. A rapid evidence synthesis. Int J Drug Policy 2014; 25 (1): 124-32.

19. French MT, Maclean JC. Underage alcohol use, delinquency, and criminal activity. Health Econ 2006; 15 (12): 1261-81.

20. Spoth R, Greenberg M, Turrisi R. Preventive interventions addressing underage drinking: state of the evidence and steps toward public health impact. Pediatrics 2008; 121 Suppl 4: S311-36.

21. Wagenaar AC, Toomey TL, Erickson DJ. Complying with the minimum drinking age: effects of enforcement and training interventions. Alcohol Clin Exp Res 2005; 29 (2): 255-62.

22. Wagenaar AC, Toomey TL. Effects of minimum drinking age laws: review and analyses of the literature from 1960 to 2000. J Stud Alcohol Suppl 2002; (14): 206-25.

23. Toomey TL, Nelson TF, Lenk KM. The age- 21 minimum legal drinking age: a case study linking past and current debates. Addiction 2009; 104 (12): 1958-65.

24. Ross HL. Confronting drunk driving: social policy for saving lives. 1992, New Haven: Yale University Press. $\mathrm{xv}, 220 \mathrm{p}$.

25. Yoruk BK, Yoruk CE. The impact of minimum legal drinking age laws on alcohol consumption, smoking, and marijuana use revisited. J Health Econ 2013; 32 (2): 477-9.

26. Zhang N, Caine E. Alcohol policy, social context, and infant health: the impact of minimum legal drinking age. Int J Environ Res Public Health 2011; 8 (9): 3796809.

27. DeJong W, Blanchette J. Case closed: research evidence on the positive public health impact of the age $21 \mathrm{mi}-$ nimum legal drinking age in the United States. J Stud Alcohol Drugs Suppl 2014; 75 Suppl 17: 108-15.

28. Wechsler H, Nelson TF. Will increasing alcohol availability by lowering the minimum legal drinking age decrease drinking and related consequences among youths? Am J Public Health 2010; 100 (6): 986-92.

29. McCartt AT, Hellinga LA, Kirley BB. The effects of minimum legal drinking age 21 laws on alcohol-related driving in the United States. J Safety Res 2010; 41 (2): 173-81.

30. Fertig AR, Watson T. Minimum drinking age laws and infant health outcomes. J Health Econ 2009; 28 (3): 73747.

31. Barreca A, Page M. A Pint for a Pound? Minimum Drinking Age Laws and Birth Outcomes. Health Econ, 2013.

32. Birckmayer J, Hemenway D. Minimum-age drinking 
laws and youth suicide, 1970-1990. Am J Public Health 1999; 89 (9): 1365-8.

33. Plunk AD, Cavazaos-Rehg P, Bierut LJ, Grucza RA. The persistent effects of minimum legal drinking age laws on drinking patterns later in life. Alcohol Clin Exp Res 2013; 37 (3): 463-9.

34. Ponicki WR, Gruenewald PJ, LaScala EA. Joint impacts of minimum legal drinking age and beer taxes on US youth traffic fatalities, 1975 to 2001. Alcohol Clin Exp Res 2007; 31 (5): 804-13.

35. Callaghan RC, Sanches M, Gatley JM, Cunningham JK. Effects of the minimum legal drinking age on alcohol-related health service use in hospital settings in Ontario: a regression-discontinuity approach. Am J Public Health 2013; 103 (12): 2284-91.

36. Callaghan RC, Sanches M, Gatley JM, Stockwell T. Impacts of drinking-age laws on mortality in Canada, 1980-2009. Drug Alcohol Depend 2014; 138: 137-45.

37. Martínez JA, Muñoz García MA, Sher KJ. A new minimum legal drinking age (MLDA)? Some findings to inform the debate. Addict Behav, 2009; 34 (4): 407-10.

38. Chile, G.d., Ley sobre Expendio y Consumo de Bebidas Alcohólicas, in 19.9252004.

39. BM. Price level ratio of PPP conversion factor (GDP) to market exchange rate. 2014. Banco Mundial (World Bank). 08 de septiembre de 2015.

40. BC. Indicadores Diarios. 2015. Banco Central de Chile. 07 de septiembre de 2015.

41. Bonnie RJ, O'Connell ME and National Research Council (U.S.). Committee on Developing a Strategy to Reduce and Prevent Underage Drinking. Reducing underage drinking: a collective responsibility. 2004, Washington,
D.C.: National Academies Press. xvi, 317 p.

42. SENDA, Décimo Estudio de Drogas en Población General. 2012.

43. Voas RB, Tippetts AS, Fell JC. Assessing the effectiveness of minimum legal drinking age and zero tolerance laws in the United States. Accid Anal Prev 2003; 35 (4): 579 87.

44. Fell JC, Fisher DA, Voas RB, Blackman K, Tippetts AS. The relationship of 16 underage drinking laws to reductions in underage drinking drivers in fatal crashes in the United States. Annu Proc Assoc Adv Automot Med 2007; 51: 537-57.

45. SENDA, Décimo Estudio de Drogas en Población Escolar. 2013.

46. Chen CM, Yi H.-y., Faden VB. Trends in Underage Drinking in the United States, 1991-2011, in Surveillance Report \#96. 2013, National Institute on Alcohol Abuse and Alcoholism Division of Epidemiology and Prevention Research Alcohol Epidemiologic Data System.

47. MINSAL, Encuesta Nacional de Salud 2009-2010. 2010.

48. MINSAL, Intervenciones Breves para recucir el consumo de alcohol de riesgo. Guía técnica para atención primaria en salud. 2011.

49. Saunders JB, Aasland OG, Babor TF, de la Fuente JR, Grant M. Development of the Alcohol Use Disorders Identification Test (AUDIT): WHO Collaborative Project on Early Detection of Persons with Harmful Alcohol Consumption-II. Addiction 1993; 88 (6): 791-804.

50. MINSAL, Estrategia Nacional sobre Alcohol. 2010.

51. MINSAL, Consumo perjudicial y Dependencia de Alcohol y otras Drogas en menores de 20 años. 2013. 